\title{
Interlayer Tuning of X-Band Frequency-Selective Surface Using Liquid Crystal
}

\author{
Amir Ebrahimi, Pouria Yaghmaee, Withawat Withayachumnankul, \\ Christophe Fumeaux, Said Al-Sarawi and Derek Abbott \\ School of Electrical and Electronic Engineering, The University of Adelaide, Adelaide, SA, 5005, Australia \\ amir.ebrahimi@adelaide.edu.au
}

\begin{abstract}
In this paper, a new concept of a voltage-controlled tunable frequency-selective surface (FSS) is introduced based on liquid crystal technology. The designed FSS consists of two periodically patterned metallic layers, separated by a thin dielectric substrate. Tunability is achieved by integrating liquid crystal cells within the substrate for each unit cell, producing interlayer capacitors. By applying a bias voltage between the front and back metallic arrays, the anisotropy axis of the liquid crystal molecules can be re-oriented, and thus the effective relative permittivity of the liquid crystals can be modified to cause a frequency shift in transmission response. Electromagnetic simulations predict $5.6 \%$ of continuous frequency tuning for this multi-layer FSS.

Index Terms - Frequency-selective surface, liquid crystal, tunable, reconfigurable.
\end{abstract}

\section{INTRODUCTION}

Various reconfigurable and tunable frequency-selective surfaces (FSS) have been developed for different frequency bands and applications. The most commonly used tuning mechanisms are, variable lumped capacitors [1], microelectromechanical (MEMs) switches [2] and tunable electromagnetic substrates (e.g. ferrite) [3]. In [4], the voltage-dependent dielectric anisotropy of liquid crystals provided a materialbased tuning mechanism for a millimetre wave FSS. Also, an infrared band tunable FSS has been reported in [5] where a liquid crystal layer was incorporated in the substrate. However, in previously reported designs, a large amount of liquid crystal was needed for tuning, resulting in high dielectric loss and costly fabrication $[4,5]$. In this paper, a new tunable doublelayer FSS is proposed based on liquid crystal. The design exploits the voltage-dependent anisotropy of the liquid crystal to vary the capacitance between the front and back FSS layers. Here, the liquid crystal cells are periodically located only at the capacitive junctions between the front and back FSS metallic layers. Taking the advantage of this new concept, the tunability can be achieved using a significantly less amount of liquid crystal which results in lower dielectric losses and decreases the material cost.

\section{LIQUid CRYSTALS MATERIAL PROPERTIES}

Liquid crystals are dielectric materials, with anisotropic characteristics that exhibit three different mesophases, between solid (crystalline) and liquid (isotropic) states, known as nematic, smetic, and cholesteric phases [6]. The nematic phase with thread or rod like molecules is the most frequently used phase, existing in both solid and liquid states. Compared to other commercially available electrically tunable materials (e.g. Barium Strontium Titanate (BST) [7]), they typically require a very low bias voltage for tuning $\left(V_{\mathrm{b}} \leq 30 \mathrm{~V}\right)$, are widely commercially available and can be integrated with flexible printing technologies [8]. The macroscopic anisotropy unit vector that points in the main average direction of the liquid crystal molecules is known as the director $\vec{n}$. The anisotropy tensor exhibits a relative permittivity $\varepsilon_{\|}$in the director direction, and $\varepsilon_{\square}$ in orthogonal directions. In a typical capacitive arrangement, where a liquid crystal cell is sandwiched between two metal electrodes, a thin layer of polyimide film $(\approx 0.4 \mu \mathrm{m})$ with fine grooves is placed on top and bottom metal layers to align the liquid crystal molecules in the unbiased state [9]. Because of these grooves, the director will be aligned in parallel with the metallic layers in the unbiased state $\left(V_{\mathrm{b}}=0 \mathrm{~V}\right)$ and the effective relative permittivity relevant for the inter-layer capacitance is $\varepsilon_{\text {eff }}=\varepsilon_{\square}$ (perpendicular state). Alternatively, when a bias voltage is applied $\left(V_{\mathrm{b}}>V_{\mathrm{th}}\right.$, where $V_{\mathrm{th}}$ corresponds to a threshold voltage), the molecules will re-orientate so that the relevant effective permittivity will gradually become the relative permittivity along the director. The final state, i.e. $\varepsilon_{\text {eff }}=\varepsilon_{\|}$, corresponds to a full alignment of the liquid crystal directors along the static electric field lines associated with the applied voltage (parallel state). The bias-dependent transition from the perpendicular to parallel state provides a continuous variation of the effective permittivity between these two states. The difference between these two extreme permittivity states can be described as $\Delta=\|-\perp$, which is commonly known as dielectric anisotropy or anisotropy of the liquid crystal. Similarly the liquid crystal dielectric tunability $(\tau)$ can be defined by $\tau=\Delta / \|$, which is the dielectric anisotropy divided by the relative permittivity along the director $\vec{n}$, and is a useful parameter to quantify the tuning capability of a given liquid crystal mixture $[10,11]$.

\section{DESIGN AND MODELLING OF THE TUNABLE FSS}

In order to demonstrate the tuning performance of the liquid crystal-based FSS, a two-layer FSS is designed to act as a simple single pole filter with an adjacent transmission zero. The front layer is composed of cross shaped metallic elements printed on one side of a thin dielectric substrate, as shown in Fig. 1. In this structure, for a $x$-polarized wave incident normally onto the FSS, the array acts as a series $L C$ resonant circuit of Fig. 1, where the inductance $L$ is mainly determined by the length $(d)$ and width $\left(a_{2}\right)$ of the cross wires and the capacitance $C$ is mainly affected by the gap between the two adjacent caps $(g)$ and their dimension $a_{l}$. Due to symmetry of the design, the array also shows the same behaviour for a $y$ polarized incident wave. 

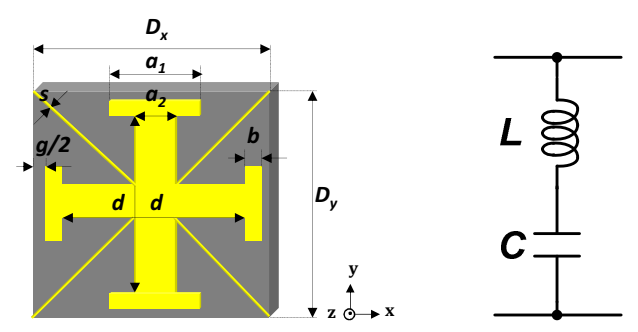

Fig. 1. Unit cell of the FSS front layer (cross shaped) and its equivalent lumped element circuit model. The dimensions are as follows: $a_{1}=1.6 \mathrm{~mm}, a_{2}=1 \mathrm{~mm}, b=0.3 \mathrm{~mm}, d=6.2 \mathrm{~mm}, s=0.3$ $\mathrm{mm}, D_{x}=D_{y}=7 \mathrm{~mm}$.

The back layer of the FSS, shown in Fig. 2, is composed of a wire grid printed on a thin dielectric substrate. It is well-known that these thin metallic strips will generate an inductive response if excited by an incident electromagnetic wave [1]. Therefore, this layer can be modelled through a lumped inductor $\left(L_{W}\right)$ as shown in Fig. 2. Finally, these two metallic arrays are separated by an intermediate dielectric layer. The middle dielectric layer should be thin enough $(\bullet 0.2 \mathrm{~mm})$ to allow strong electromagnetic interaction between the front and the back arrays. This very thin intermediate dielectric layer will create an interlayer capacitor wherever the front cross shape resonators and the back wire grid overlap in the overall configuration as shown in Fig. 3. The tuning characteristic of this FSS is achieved by controlling this interlayer capacitance value $\left(C_{\text {int }}\right)$. In this work, in order to tune the FSS, the liquid crystal is introduced in cells created in the substrate at the location of the capacitors. As in [12], thin conductor strips with a width $s$ are added in the design to electrically connect every unit cell for facilitating application of an external bias voltage (see Fig. 1). Due to the very narrow width of the conductor strips, they show a very large inductance which is negligible at high frequencies compared with $L$ and $L_{W}$ and hence their effect on the transmission response is minimal.
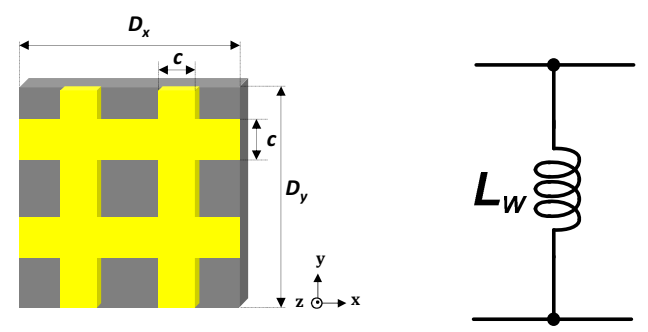

Fig. 2. Unit cell of the FSS back layer (wire grid) and its equivalent lumped element circuit model. The dimensions are as follows: $c=1$ $\mathrm{mm}, D_{x}=D_{\mathrm{y}}=7 \mathrm{~mm}$.

Varying the bias voltage changes the effective permittivity of the liquid crystal, and hence causes a change in interlayer capacitor value. The change in the capacitance leads to a frequency shift in the transmission response of the FSS. The final schematic of the FSS unit cell is shown in Fig. 3, where the location of the liquid crystal cells are visible in the overlap areas of the front and back metallic arrays.

To gain better insight into the qualitative FSS behavior, a lumped element equivalent circuit is developed to describe the expected frequency response of the entire structure. As shown in Fig. 4, the equivalent model is composed of two parallel branches representing the two array layers. The left branch of the circuit models the cross-shaped metallic array that shows a band-stop behaviour. The capacitor in this branch is a function of the gap between two adjacent cross elements $(g)$. The $L_{l}$ is a portion of inductor $L$ before the center of the overlap capacitor

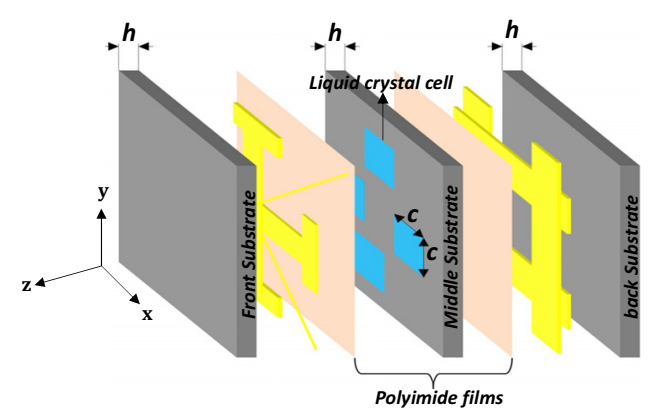

Fig. 3. Single unit cell of the multi-layered FSS structure, illustrating each individual layer and the liquid crystal sample placed in the middle layer, in overlapping areas of the front pattern with the back copper grid. The dimensions are as follows: $c=1 \mathrm{~mm}$ and $h=0.127$ $\mathrm{mm}$.

and $L_{2}$ models the portion which is located after the center of overlap capacitor. Their value is a function of $a_{2}$ and $d$ (Fig. 1). The right branch, on the other hand, models the back side of the FSS, which shows a purely inductive behaviour and has a band-pass response. Finally the interlayer capacitor is modelled by a lumped voltage dependent capacitor $C_{\text {int }}$, which can be tuned by applying a voltage between the metallic elements of the two array layers. Further, in the circuit model, the substrates are modelled by three short pieces of transmission lines having the characteristic impedance of $Z_{0} / \sqrt{\varepsilon_{\mathrm{r}}}$, where, is the relative permittivity of the thin substrate layers. The free spaces on the either sides of the FSS are modelled with a characteristic impedance of $Z_{0}$. In this circuit, all metallic and dielectric losses are ignored for simplicity.

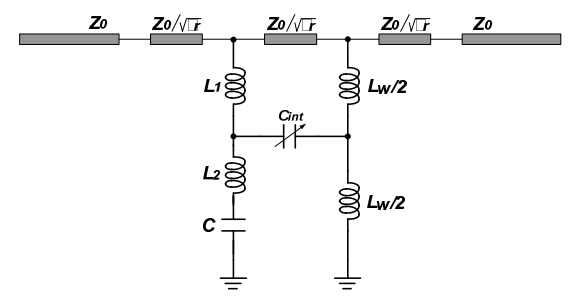

Fig. 4. Lumped element circuit model of the designed tunable FSS Elements values are: $L_{1}=0.76 \mathrm{nH}, L_{2}=0.38 \mathrm{nH}, C=0.094 \mathrm{pF}, L_{W}=$ $0.58 \mathrm{nH}$, and $C_{\text {int }}=0.1 \mathrm{pF}$.

\section{RESULTS AND DISCUSSIONS}

The unit cell of the frequency selective surface is simulated in CST Microwave Studio, using the frequency-domain solver over a frequency range of $6-15 \mathrm{GHz}$. For this full-wave electromagnetic simulation the front, middle and back dielectric layers are simulated as a Rogers Duroid 5880 substrate $\left(\varepsilon_{r}=2.20, \tan \delta=0.0009\right.$, thickness $\left.h=0.127 \mathrm{~mm}\right)$. Also, the lumped element circuit model is developed in Advanced Design System (ADS) software using the first-order element values approximation presented in [13] and with the values selected to best fit the full wave simulations. The continuous frequency tuning of the FSS is studied using three different liquid crystal samples, known as K15 (5CB), BL037 and the new GT3-23001 mixture, with corresponding dielectric anisotropy $(\Delta \square)$ of $0.18,0.26$ and 0.80 respectively. The anisotropy of the liquid crystal is taken into account by considering the two permittivity tensor values in the extreme states ( $\square_{\perp}$ and $\square_{\|}$), with their corresponding loss tangent (tan $\delta_{\perp}$ and $\left.\tan \delta_{\|}\right)$, given in Table I $[10,14]$. The full-wave electromagnetic simulations indicate that by varying the effective relative permittivity of the liquid crystal for the K15 sample from $\varepsilon_{\text {eff }}=2.72$ to 2.90 , the pass-band center frequency of the designed FSS shifts from 9.32 to $9.20 \mathrm{GHz}(120 \mathrm{MHz})$ 
(Fig. 5 (a)). This is equivalent to a frequency shift of $1.3 \%$ relative to the lower frequency (Fig. 5 (a)). However, when the BL037 liquid crystal sample is used, the frequency shifts from 9.50 to $9.29 \mathrm{GHz}$, indicating a frequency shift of around $2.2 \%$ i.e. approximately twice the frequency tuning obtained with the initial sample (K15) (Fig. 5 (b)). Finally, when the cells are filled with the GT3-23001 liquid crystal mixture from Merck, the pass-band center frequency of the structure shifts from 9.49 to $8.98 \mathrm{GHz}(510 \mathrm{MHz})$, which corresponds to the larger frequency shift of $5.6 \%$ (Fig. 5 (c)).

TABLE I

PROPERTIES OF LIQUid CRYSTALS AT ROOM TEMPERATURE 20 C

\begin{tabular}{|l|c|c|l|l|l|l|}
\hline samples & $\perp$ & $\|$ & $\tan \boldsymbol{\delta}_{\perp}$ & $\tan \boldsymbol{\delta}_{\|}$ & \multicolumn{1}{|c}{$\boldsymbol{\Delta}$} & freq. \\
\hline $\begin{array}{l}\mathrm{K} 15 \\
(5 \mathrm{CB})\end{array}$ & 2.72 & 2.90 & 0.03 & 0.03 & 0.18 & $\begin{array}{l}1-10 \\
\mathrm{GHz}\end{array}$ \\
\hline BL037 & 2.35 & 2.61 & 0.06 & 0.06 & 0.26 & $\begin{array}{l}1-10 \\
\mathrm{GHz}\end{array}$ \\
\hline $\begin{array}{l}\mathrm{GT3}- \\
23001\end{array}$ & 2.50 & 3.30 & 0.0143 & 0.0038 & 0.80 & $\begin{array}{l}19 \\
\mathrm{GHz}\end{array}$ \\
\hline
\end{tabular}

In the ADS circuit simulations corresponding to the three cases above, the value of $C_{\text {int }}$ was modified based on variation of $\varepsilon_{\text {eff. }}$ These results illustrate that $C_{\text {int }}$ changes from 0.088 to $0.093 \mathrm{pF}$ for K15, 0.080 to $0.090 \mathrm{pF}$ for BL037 and from 0.081 to $0.106 \mathrm{pF}$ for GT3-2300, for both perpendicular and parallel states respectively as shown in Table II.

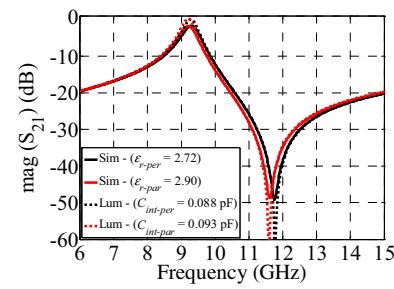

(a)

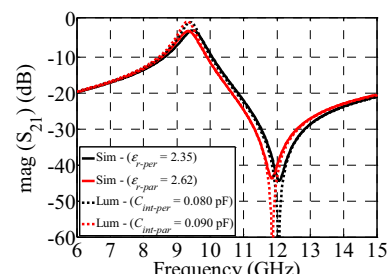

(b)

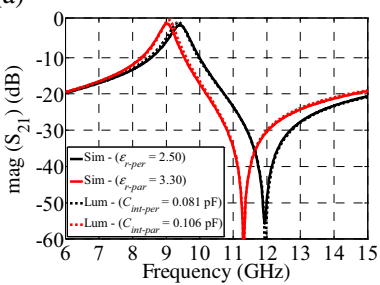

(c)

Fig. 5 Frequency tuning range and insertion loss comparison for, (a) K15 (5CB) (c) BL037 and (b) GT3-23001.

TABLE II

COMPARISON OF Full-WAVE SiMULATION AND LUMPED-ElEMENT SIMULATION FOR K15, BL037 AND GT3-23001

\begin{tabular}{|c|c|c|c|c|c|c|c|c|}
\hline Models & Sim. & $\begin{array}{c}\mathrm{f} \perp \text {, zero } \\
(\mathrm{GHz})\end{array}$ & $\begin{array}{c}\mathrm{f}_{\|, \text {zero }} \\
(\mathrm{GHz})\end{array}$ & $\begin{array}{c}\mathrm{f} \perp \text {, pole } \\
(\mathrm{GHz})\end{array}$ & $\begin{array}{l}f_{\|, \text {pole }} \\
(\mathrm{GHz})\end{array}$ & $\begin{array}{l}\mathrm{C}_{\text {int, }} \perp \\
(\mathrm{pF})\end{array}$ & $\begin{array}{l}\mathrm{C}_{\text {int, }} \| \\
(\mathrm{pF})\end{array}$ & $\begin{array}{l}\text { Tun. } \\
(\%)\end{array}$ \\
\hline \multirow[t]{2}{*}{ K15 } & CST & \multirow{2}{*}{11.76} & \multirow{2}{*}{11.61} & \multirow{2}{*}{9.32} & \multirow{2}{*}{9.20} & & & \multirow{2}{*}{1.3} \\
\hline & ADS & & & & & 0.088 & 0.093 & \\
\hline \multirow[t]{2}{*}{ BL037 } & CST & \multirow{2}{*}{12.06} & \multirow{2}{*}{11.85} & \multirow{2}{*}{9.50} & \multirow{2}{*}{9.29} & & & \multirow{2}{*}{2.2} \\
\hline & ADS & & & & & 0.080 & 0.090 & \\
\hline \multirow{2}{*}{$\begin{array}{l}\text { GT3- } \\
23001\end{array}$} & CST & \multirow{2}{*}{11.94} & \multirow{2}{*}{11.31} & \multirow{2}{*}{9.49} & \multirow{2}{*}{8.98} & & & \multirow{2}{*}{5.6} \\
\hline & ADS & & & & & 0.081 & 0.106 & \\
\hline
\end{tabular}

The obtained information will be used to demonstrate experimentally a tunable FSS and the principle can be scaled for operation at higher frequencies, e.g. in the Ka-band, where the dissipation of liquid crystal is lower and lumped variable capacitors become too bulky for integration into the FSS.

\section{CONCLUSION}

A new concept of liquid crystals based tunable FSS has been proposed which provides frequency tuning using a small amount of liquid crystals. The principle and operation have been studied through full wave and lumped element circuit model simulations. The performance of the proposed tuning method has been investigated using three different types of liquid crystals predicting a maximum tuning range of $5.6 \%$ with GT3-23001 type liquid crystals.

\section{ACKNOWLEDGMENT}

The authors acknowledge the support of the Australian Research Council (ARC) under the Discovery Project DP120100661 and also Merck KGaA, Germany for providing the liquid crystal samples.

\section{REFERENCES}

[1] F. Bayatpur and K. Sarabandi, "Tuning performance of metamaterial-based frequency selective surfaces," IEEE Transactions on Antennas and Propagation, vol. 57, pp. 590592, 2009.

[2] B. Schoenlinner, A. Abbaspour-Tamijani, L. C. Kempel, and G. M. Rebeiz, "Switchable low-loss RF MEMS Ka-band frequencyselective surface," IEEE Transactions on Microwave Theory and Techniques, vol. 52, pp. 2474-2481, 2004.

[3] T. K. Chang, R. J. Langley, and E. A. Parker, "Frequency selective surfaces on biased ferrite substrates," Electronics Letters, vol. 30, pp. 1193-1194, 1994.

[4] H. Wenfei, R. Dickie, R. Cahill, H. Gamble, Y. Ismail, V. Fusco, D. Linton, N. Grant, and S. Rea, "Liquid crystal tunable $\mathrm{mm}$ wave frequency selective surface," IEEE Microwave and Wireless Components Letters, vol. 17, pp. 667-669, 2007.

[5] J. A. Bossard, L. Xiaotao, L. Ling, Y. Seokho, D. H. Werner, B. Weiner, T. S. Mayer, P. F. Cristman, A. Diaz, and I. C. Khoo, "Tunable frequency selective surfaces and negative-zero-positive index metamaterials based on liquid crystals," IEEE Transactions on Antennas and Propagation, vol. 56, pp. 13081320, 2008.

[6] S. Mueller, A. Penirschke, C. Damm, P. Scheele, M. Wittek, C. Weil, and R. Jakoby, "Broad-band microwave characterization of liquid crystals using a temperature-controlled coaxial transmission line," IEEE Transactions on Microwave Theory and Techniques, vol. 53, pp. 1937-1945, 2005.

[7] M. Sazegar, Z. Yuliang, H. Maune, C. Damm, Z. Xianghui, and R. Jakoby, "Compact tunable phase shifters on screen-printed BST for balanced phased arrays," IEEE Transactions on Microwave Theory and Techniques, vol. 59, pp. 3331-3337, 2011.

[8] P. Yaghmaee, A. K. Horestani, B. Bates, and C. Fumeaux, "A multi-layered tunable stepped-impedance resonator for liquid crystal characterization," Proceedings of Asia-Pacific Microwave Conference, pp. 776-778, 2012.

[9] Y. Garbovskiy, L. Reisman, Z. Celinski, R. E. Camley, and A. Glushchenko, "Metallic surfaces as alignment layers for nondisplay applications of liquid crystals," Applied Physics Letters, vol. 98, pp. 73301-3, 2011.

[10] P. Yaghmaee, C. Fumeaux, B. Bates, A. Manabe, O. H. Karabey, and R. Jakoby, "Frequency tunable S-band resonator using nematic liquid crystal," IET Electronics Letters, vol. 48, pp. 798800, 2012.

[11] S. Mueller, M. Koeberle, F. Goelden, A. Penirschke, A. Gaebler, A. Lapanik, W. Haase, and R. Jakoby, "W-band characterization of anisotropic liquid crystals at room temperature," 38th European Microwave Conference, pp. 119-122, 2008.

[12] W. Withayachumnankul, C. Fumeaux, and D. Abbott, "Planar array of electric-LC resonators with broadband tunability," IEEE Antennas and Wireless Propagation Letters, vol. 10, pp. $577-$ 580, 2011.

[13] W. R. Smythe and W. R. Smythe, "Static and dynamic electricity," McGraw-Hill Book Company Inc., 1950.

[14] P. Yaghmaee, W. Withayachumnankul, A. K. Horestani, A. Ebrahimi, B. Bates and C. Fumeaux, "Tunable electric-LC resonators using liquid crystal," IEEE International Symposium on Antenna and Propagation APSURSI, 2013. 